\title{
Transtorno obsessivo-compulsivo em tempos de pandemia de COVID-19
}

\author{
Obsessive-compulsive disorder in the COVID-19 pandemic \\ Trastorno obsesivo-compulsivo en tiempos pandemicos de COVID-19
}

Recebido: 09/05/2021 | Revisado: 13/05/2021 | Aceito: 13/05/2021 | Publicado: 15/05/2021

\author{
Lúcia Gabriela Costa Silva \\ ORCID: https://orcid.org/0000-0002-7523-1204 \\ Universidade Tiradentes, Brasil \\ E-mail: gabycostaandrade@gmail.com \\ Juliana Leal Freitas Maia \\ ORCID: https://orcid.org/0000-0002-1751-4572 \\ Universidade Tiradentes, Brasil \\ E-mail: juliana.maia@ souunit.com.br
}

\begin{abstract}
Resumo
O presente estudo é uma pesquisa bibliográfica com o objetivo de analisar as mudanças sofridas nos pacientes com Transtorno Obsessivo-compulsivo (TOC) em tempos de pandemia de COVID-19, por meio da revisão de artigos científicos disponíveis na base de dados Pubmed utilizando os descritores "Obsessive-compulsive disorder" e "COVID-19" publicados no período de janeiro de 2020 a abril de 2021. A reflexão realizada com base na literatura revelou o aumento e alteração na gravidade dos sintomas dos pacientes com TOC, especialmente naqueles que sofrem obsessão de contaminação e compulsão de lavagem. Dos pacientes que apresentaram exacerbação dos sintomas, essa foi associada a uma piora nos sintomas de estresse, ansiedade e depressão. Além disso, foram constatadas, nesse período pandêmico, manifestações de sintomas obsessivos-compulsivos na população geral. Portanto, fazem-se necessárias pesquisas futuras pós Covid-19 para avaliar como evoluirá o quadro clínico dos pacientes que apresentaram exacerbação do TOC, bem como para determinar se os novos sintomas de TOC na população geral estão relacionados a um diagnóstico definitivo de TOC, a uma expressão de ansiedade proporcionada pela pandemia ou a uma combinação de ambos.
\end{abstract}

Palavras-chave: Transtorno obsessivo-compulsivo (TOC); Pandemia; COVID-19.

\begin{abstract}
The present study is bibliographical research with the objective of analyzing the changes suffered by Obsessivecompulsive Disorder (OCD) patients in the COVID-19 pandemic through the review of scientific articles available in the Pubmed database, chosen using the descriptors "Obsessive-compulsive disorder" and "COVID-19", published from January 2020 to April 2021. The analysis based on the literature revealed the increase and change in the severity of the symptoms of OCD patients, especially in those who have contamination obsession and washing compulsion. The exacerbation of symptoms in patients was associated with a worsening of stress, anxiety and depression traits. Furthermore, it was observed the manifestation of obsessive-compulsive symptoms in the general population in that period. Therefore, future post-Covid-19 research is needed to assess how the clinical picture of patients with OCD exacerbation will evolve, as well as to determine whether new OCD symptoms in the general population are related to a definitive diagnosis of OCD, an expression of anxiety caused by the pandemic or a combination of both.
\end{abstract}

Keywords: Obsessive-compulsive disorder (OCD); Pandemic; COVID-19.

\begin{abstract}
Resumen
El presente estudio es una investigación bibliográfica con el objetivo de analizar los cambios sufridos en pacientes con Trastorno Obsesivo-Compulsivo (TOC) en tiempos de la pandemia COVID-19, mediante la revisión de artículos científicos disponibles en la base de datos Pubmed utilizando los descriptores "Obsesivo -trastorno compulsivo" y "COVID-19" publicados entre enero de 2020 y abril de 2021. La reflexión realizada a partir de la literatura reveló el aumento y alteración de la gravedad de los síntomas de los pacientes con TOC, especialmente en aquellos que padecen obsesión por la contaminación y la compulsión al lavado. De los pacientes que experimentaron una exacerbación de los síntomas, esto fue asociado con un empeoramiento de los síntomas de estrés, ansiedad y depresión. Además, durante este período pandémico, fueron encontradas manifestaciones de síntomas obsesivocompulsivos en la población general. Por lo tanto, se necesita una investigación futura posterior a Covid-19 para evaluar cómo evolucionará el cuadro clínico de los pacientes con exacerbación del TOC, así como para determinar si los nuevos síntomas del TOC en la población general están relacionados con un diagnóstico definitivo de TOC, la expresión de ansiedad provocada por la pandemia o una combinación de ambos.
\end{abstract}

Palabras clave: Trastorno obsesivo-compulsivo (TOC); Pandemia; COVID-19. 


\section{Introdução}

O transtorno obsessivo-compulsivo (TOC) é caracterizado por pensamentos, imagens ou impulsos indesejáveis e angustiantes (obsessões) e/ou comportamentos repetitivos ou atos mentais (compulsões), os quais o indivíduo se sente impulsionado a realizar, seja em relação à obsessão ou de acordo com regras rígidas que ele acredita que devem ser aplicadas de forma inflexível ou para alcançar uma sensação de completude como conceitua Silva et al. (2021). Trata-se de uma condição debilitante, que diminui a qualidade de vida, associada a um imenso sofrimento, que atinge até $3.1 \%$ da população geral, como aponta Rajkumar (2020).

De acordo com Ornell et al. (2021), obsessões ou compulsões consomem tempo (por exemplo, demoram mais de uma hora por dia) e causam sofrimento e prejuízo nas áreas sociais, profissionais e outras áreas importantes da vida de um indivíduo, sendo essas as características essenciais para diagnosticar a doença.

Obsessões e compulsões variam amplamente entre os indivíduos, porém, existem as "dimensões dos sintomas" que são temas identificáveis, variáveis e incluem: a dimensão de limpeza com presença de medo de contaminação e rituais de limpeza; a de simetria com obsessões de simetria e compulsões de repetição, ordenação e contagem; a de pensamentos proibidos ou tabu, a exemplos dessa, incluem obsessões agressivas, sexuais e religiosas e compulsões relacionadas à temática, e à dimensão de danos associada a pensamentos ou imagens sobre danos que acontecem a si mesmo ou a outros e geram compulsões de verificação, afirma Aardema (2020).

Embora as dimensões de sintomas indiquem que, de certa forma, o TOC seja aparentemente uma desordem homogênea, os sintomas apresentados no transtorno são heterogêneos, podendo se apresentar inclusive com uma gama de sintomas menos comuns, incluindo escrupulosidade, ciúme obsessivo e obsessões musicais, como descreve Chakraborty e Karmakar (2020). Do múltiplo domínios de sintomas, de acordo com Prestia et al. (2020), as obsessões de contaminação e lavagem compulsiva das mãos estão entre os mais comuns, afetando cerca de 50\% dos pacientes.

A COVID-19 é uma doença infecciosa causada pelo vírus SARS-CoV-2, relacionado à síndrome respiratória aguda, que emergiu no final de 2019 em Wuhan, Província de Hubei, China, rapidamente se disseminou por todos os continentes, aumentando exponencialmente o número de infectados e ocasionando milhares de mortes no mundo, de acordo com Tanir et al. (2020). Nesse contexto, em 11 de março de 2020, o surto mundial de COVID-19 foi classificado como uma pandemia pela Organização Mundial da Saúde. A fim de limitar a propagação do vírus SARS-CoV-2, muitos países ao redor do mundo tomaram medidas rígidas, como colocar cidades inteiras em quarentena, com milhares de pessoas vivendo em confinamento, conforme constata Benatti et al. (2020).

Sabe-se que as pandemias têm impacto não apenas no contexto biológico e social, mas também no psicológico (Benatti et al., 2020). Durante surtos anteriores, como o da Síndrome Respiratória Grave Aguda (SARS), Síndrome Respiratória do Oriente Médio (MERS) e Influenza foram documentados exarcebações da sintomatologia dos pacientes com transtorno obsessivo- compulsivo, conforme aduz Storch et al. (2020).

Nessa esteira, a pandemia COVID-19 está inquestionavelmente impactando na saúde mental da população em todo o mundo. O medo da contaminação pode aumentar os níveis de estresse em indivíduos saudáveis e intensificar os sintomas psiquiátricos em pacientes com doenças pré-existentes, especialmente o transtorno obsessivo-compulsivo (Matsunaga et al., 2020). No entanto, o alto nível de heterogeneidade e seletividade entre os subtipos de sintomas é incongruente com a noção de que COVID-19 é sempre diretamente relevante para o TOC. É bem possível que os efeitos negativos da atual pandemia sejam mais fortes em alguns indivíduos com TOC, principalmente, nos que apresentam obsessões relacionadas a danos, medo de contaminação e compulsões por limpeza, como descrito por Aardema (2020).

Diante desse quadro pandêmico, os indivíduos têm tomado medidas para se protegerem, aderindo às diretrizes oficiais de segurança delineadas por órgãos governamentais e de saúde pública, que incluem distanciamento social, uso de máscaras 
faciais, prática de higiene adequada e manutenção de outros comportamentos preventivos (Storch et al., 2021).

Nesse contexto, para Ornell et al. (2021), o risco iminente de contaminação cria uma necessidade lógica de autovigilância e hábitos de higiene. No entanto, esse tipo de informação pode ter implicações drásticas para sujeitos com TOC, especialmente aqueles que têm obsessão por contaminação e compulsão por lavar, uma vez que distorções cognitivas e estratégias compensatórias (rituais de limpeza) não são mais irracionais ou superdimensionadas, ao contrário, essas ideias tornam-se necessárias, legítimas e socialmente aceitas, gerando validação plausível para a intensificação da compulsão e rituais de limpeza.

Segundo Shafran et al. (2020), jovens e adultos suscetíveis ao desenvolvimento de transtorno obsessivo-compulsivo provavelmente serão profundamente afetados pela atual pandemia de COVID-19, tendo em vista que o conteúdo das obsessões não é aleatório, são desencadeados por eventos externos e estão relacionados a preocupações atuais. Nesse sentido, o surto atual de COVID-19 representa um desafio único tanto para pacientes com TOC, devido a um aumento potencial na frequência de obsessões e compulsões naqueles mais suscetíveis, como para psiquiatras, que encontram um desafio na avaliação para diagnóstico de TOC, haja vista que a ansiedade decorrente da pandemia pode proporcionar sintomas obsessivos, e esses não serem necessariamente decorrentes de TOC, como conceitua Benatti et al. (2020).

Como descrito por Perkes et al. (2020), pessoas com transtorno obsessivo-compulsivo podem ter uma preocupação desproporcional ou irracional em relação ao risco de se infectarem ou responsabilidade pela transmissão infecciosa, o que pode desencadear nesses indivíduos uma compulsão em lavagem excessiva das mãos e prevenção significativa de contaminantes potenciais. A exposição aos medos que geram a obsessão é parte essencial do tratamento do TOC, no entanto, no período pandêmico atual, tal medida é contraindicada, uma vez que pode expor o paciente a um risco evitável. Portanto, é importante explorar os efeitos da pandemia em pessoas com TOC para entender a melhor forma de avaliar, diagnosticar e gerenciar a condição em tempos de COVID-19.

\section{Metodologia}

Trata-se de um estudo que tem como base a análise, por revisão bibliográfica, de artigos científicos disponíveis na base de dados Pubmed publicados no período de janeiro de 2020 a abril de 2021, que compreende o período sob efeito da atual pandemia de COVID-19. Os descritores utilizados foram Obsessive-compulsive disorder e COVID-19, consultados através dos Descritores em Ciências da Saúde (DeSCS). A busca dos materiais que abordaram a temática em questão resultou em um total de 108 estudos potencialmente elegíveis. Um registro adicional foi obtido por meio da busca manual das listas de referência dos artigos incluídos e das publicações referentes ao Transtorno obsessivo-compulsivo (TOC). Após a aplicação dos critérios de inclusão e exclusão, foram selecionados 30 artigos.

A presente revisão possui uma abordagem qualitativa de acordo com Pereira et al. (2018) por se tratar de uma síntese de estudos relacionado ao tema principal, com interpretação e agrupamento dos resultados de outros autores mas sem a aplicação de análise estáticas de forma integral. O dados foram analisados por meio da técnica de análise de conteúdo em três etapas: a pré análise, com a busca e seleção dos artigos na base de dados de escolha, a extração dos dados e o tratamento e a interpretação dos resultados obtidos.

Os critérios utilizados para inclusão de um artigo nesta revisão foram estudos publicados no período de janeiro de 2020 a abril de 2021, que abordassem a temática em comento - a relação entre Transtorno obsessivo-compulsivo e a pandemia de Covid-19. Outros critérios de inclusão foram populações de todas as faixas etárias, sem restrição de localidade e artigos publicados nos idiomas inglês, português e espanhol. Foram excluídos artigos duplicados, não relacionados à temática proposta e que não atendessem aos critérios de inclusão da pesquisa. 


\section{Resultados}

Após a aplicação dos critérios de inclusão e exclusão pré-determinados, foram selecionados 30 artigos, dos quais 15 $\operatorname{artigos}(50 \%)$ apresentavam um enfoque quantitativo, 9 artigos (30\%) utilizavam uma abordagem quali-quantitativa e 6 artigos (20\%) apresentavam um enfoque qualitativo.

O Quadro 1 apresenta distribuição das publicações segundo autor, país de origem, objetivos, métodos e resultados/conclusão. Verificou-se que o continente asiático é o que produz mais publicações associadas ao tema, com 40\% dos trabalhos. Outro fator a ressaltar é a diversidade de instrumentos validados empregados na avaliação dos pacientes com TOC no período da pandemia, sendo o instrumento mais utilizado a Escala Yale-Brown Obsessivo-Compulsiva.

Quadro 1 - Resultados e Objetivos dos Trabalhos Estudados.

\begin{tabular}{|c|c|c|c|}
\hline AUTOR (ES) & PAIS & OBJETIVO & RESULTADOS/CONCLUSÃO \\
\hline $\begin{array}{l}\text { Aardena } \\
\text { et al. }(2020)\end{array}$ & Canadá & $\begin{array}{l}\text { Explorar os temores de } \\
\text { contaminação nos paci- } \\
\text { entes com TOC no con- } \\
\text { texto da atual pandemia } \\
\text { de COVID-19. }\end{array}$ & $\begin{array}{l}\text { Conclui que há uma forte probabilidade que os } \\
\text { efeitos negativos da atual pandemia sejam mais } \\
\text { fortes em alguns indivíduos com TOC, } \\
\text { principalmente, nos que apresentam obsessões } \\
\text { relacionadas a danos, medo de contaminação e } \\
\text { compulsões por limpeza. Com a ressalva de que } \\
\text { não se pode generalizar a todas as dimensões ou } \\
\text { casos concretos. }\end{array}$ \\
\hline Abba-Aji et al. (2020) & Canadá & $\begin{array}{l}\text { Analisar a presença de } \\
\text { sintomas obsessivos e } \\
\text { compulsivos na população } \\
\text { geral durante a fase inicial } \\
\text { da COVID-19. }\end{array}$ & $\begin{array}{l}\text { Os resultados demonstram que a maioria dos } \\
\text { entrevistados apresentavam obsessões } \\
\text { relacionadas à contaminação e compulsões de } \\
\text { lavagem, ambas iniciadas durante a pandemia. Os } \\
\text { indivíduos que apresentaram novos sintomas } \\
\text { obsessivos e compulsivos foram mais propensos a } \\
\text { ter moderado/alto estresse, Transtorno de } \\
\text { Ansiedade Generalizada e Transtorno Depressivo } \\
\text { durante a pandemia. }\end{array}$ \\
\hline Alhusseini et al. (2021) & $\begin{array}{l}\text { Arábia } \\
\text { Saudita }\end{array}$ & $\begin{array}{l}\text { Avaliar a frequência de } \\
\text { depressão e TOC entre a } \\
\text { população em geral } \\
\text { durante a pandemia de } \\
\text { COVID-19 }\end{array}$ & $\begin{array}{l}\text { O artigo evidenciou que o período de pandemia } \\
\text { COVID-19 está associado a riscos significativos } \\
\text { para a saúde mental. Nesse ínterim, foi } \\
\text { identificado que os homens, idosos, casados, os } \\
\text { baixos níveis socioeconômicos, desempregados e } \\
\text { de alto nível educacional correm um risco maior } \\
\text { de desenvolver TOC, nesse período de pandemia. }\end{array}$ \\
\hline Benatti et al. (2020) & Itália & $\begin{array}{l}\text { Avaliar o impacto da } \\
\text { pandemia de COVID-19 } \\
\text { em pacientes com TOC. }\end{array}$ & $\begin{array}{l}\text { Percebeu-se uma piora clínica dos pacientes com } \\
\text { TOC, havendo um surgimento significativo de } \\
\text { novas obsessões e fenótipos de compulsões } \\
\text { juntamente com exacerbação das compulsões } \\
\text { passadas. Além disso, evidenciou um crescimento } \\
\text { de comportamento de evitação, ideação suicida, } \\
\text { de verificação na Internet, distúrbios do sono e } \\
\text { dificuldades de trabalho. }\end{array}$ \\
\hline $\begin{array}{l}\text { Chakraborty e Karmakar } \\
\qquad(2020)\end{array}$ & Irã & $\begin{array}{l}\text { Avaliar o efeito do CO- } \\
\text { VID- } 19 \text { em pacientes com } \\
\text { TOC. }\end{array}$ & $\begin{array}{l}\text { O estudo concluiu que não houve nenhum au- } \\
\text { mento significativo dos sintomas de TOC durante } \\
\text { a pandemia de COVID-19. Os pacientes que } \\
\text { foram previamente diagnosticados com TOC de } \\
\text { obsessão de contaminação e lavagem compulsiva } \\
\text { não mostraram qualquer aumento em seu } \\
\text { comportamento de lavar. }\end{array}$ \\
\hline Jelinek et al. (2021) & Alemanha & $\begin{array}{l}\text { Avaliar mudança na } \\
\text { gravidade dos sintomas de } \\
\text { TOC, as razões para essa, } \\
\text { bem como as crenças e } \\
\text { experiências dos } \\
\text { participantes associadas } \\
\text { ao COVID-19. }\end{array}$ & $\begin{array}{l}\text { O artigo constatou que a maioria dos participantes } \\
\text { com TOC foi afetada negativamente pela } \\
\text { pandemia COVID-19. Nessa esteira, observou-se } \\
\text { um aumento dos sintomas do TOC mais } \\
\text { significativos em pacientes lavadores, a piora foi } \\
\text { associada principalmente à redução da } \\
\text { mobilidade, conflitos interpessoais, fatores } \\
\text { econômicos e as crenças disfuncionais relaci- } \\
\text { onadas à higiene. }\end{array}$ \\
\hline
\end{tabular}




\begin{tabular}{|c|c|c|c|}
\hline Ji G et al. (2020) & China & $\begin{array}{l}\text { Investigar se o medo de } \\
\text { COVID-19 afeta a pre- } \\
\text { valência de possível TOC. }\end{array}$ & $\begin{array}{l}\text { O artigo verificou que o nível de ansiedade, de } \\
\text { quarentena e a intensidade do medo de COVID- } \\
19 \text { foram significativamente mais baixos nas } \\
\text { pesquisas } 2 \text { e } 3 \text { do que na pesquisa } 1 \text {. Esses } \\
\text { resultados sugeriram que as interaçóes entre } \\
\text { medo, ansiedade e a quarentena induzida são } \\
\text { fatores de risco para um aumento no escore Y- } \\
\text { BOCS. }\end{array}$ \\
\hline Kaveladze et al. (2021) & $\begin{array}{l}\text { Estados } \\
\text { Unidos }\end{array}$ & $\begin{array}{l}\text { Verificar alterações da } \\
\text { dimensão dos sintomas de } \\
\text { TOC desde o início da } \\
\text { pandemia em pessoas } \\
\text { diagnosticados ou que } \\
\text { suspeitavam possuir TOC. }\end{array}$ & $\begin{array}{l}\text { O artigo concluiu que a maioria dos participantes } \\
\text { apresentaram piora de seus sintomas de TOC } \\
\text { desde o início da pandemia. Embora a piora } \\
\text { diferisse entre as dimensões dos sintomas, } \\
\text { notavelmente, os de simetria e completude tinham } \\
\text { menos probabilidade de piorar do que outros. }\end{array}$ \\
\hline Khosravani et al. (2021a) & Irã & $\begin{array}{l}\text { Comparar um grupo de } \\
\text { pacientes com TOC antes } \\
\text { e durante o COVID-19, } \\
\text { em relação as alterações } \\
\text { na gravidade e nas } \\
\text { dimensões dos sintomas. }\end{array}$ & $\begin{array}{l}\text { No artigo, foi observado um aumento do so- } \\
\text { frimento dos pacientes com TOC, devido à } \\
\text { ameaça e ao medo de ser contaminado, dos } \\
\text { efeitos socioeconômicos da pandemia, dos } \\
\text { estrangeiros (xenofobia) diante à possibilidade de } \\
\text { esses estarem infectados e devido aos sintomas de } \\
\text { estresse traumático e comportamentos } \\
\text { compulsivos de verificação. }\end{array}$ \\
\hline Khosravani et al. (2021b) & Irã & $\begin{array}{l}\text { Determinar o efeito da } \\
\text { pandemia de COVID-19 } \\
\text { na gravidade, nas di- } \\
\text { mensões dos sintomas } \\
\text { TOC, bem como, os } \\
\text { efeitos das respostas ao } \\
\text { estresse nesses pacientes. }\end{array}$ & $\begin{array}{l}\text { O resultado demostrou que a COVID-19 está } \\
\text { relacionada a um aumento em todas as dimensões } \\
\text { dos sintomas de TOC, incluindo contaminação, } \\
\text { responsabilidade pelo dano, pensamentos } \\
\text { inaceitáveis e simetria. Além disso, foi percebido } \\
\text { um aumento a gravidade geral do TOC. }\end{array}$ \\
\hline Littman et al. (2020) & Israel & $\begin{array}{l}\text { Avaliar a mudança dos } \\
\text { sintomas de TOC em } \\
\text { resposta ao COVID-19 } \\
\text { durante o período de } \\
\text { quarentena. }\end{array}$ & $\begin{array}{l}\text { O estudo concluiu que a maioria dos pacientes } \\
\text { com TOC não foi afetada pela crise COVID-19, } \\
\text { haja vista que, não houve na maioria dos } \\
\text { entrevistados, diferença na gravidade dos } \\
\text { sintomas, tampouco na transição de suas ca- } \\
\text { tegorias. }\end{array}$ \\
\hline Matsunaga et al. (2020) & Japão & 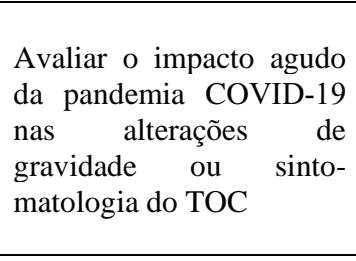 & $\begin{array}{l}\text { Os resultados apontaram que somente } 10 \% \text { da } \\
\text { amostra experimentaram a deterioração da } \\
\text { gravidade dos sintomas obsessivos e compul- } \\
\text { sivos, sendo afetados principalmente indivíduos } \\
\text { com sintomas associados à contamina- } \\
\text { ção/lavagem. Esses foram mais propensos a ter } \\
\text { maior traço de ansiedade e estado depressivo }\end{array}$ \\
\hline Mrklas et al. (2020) & Canadá & $\begin{array}{lr}\text { Avaliar a prevalência } \\
\text { autorreferida de estresse, } \\
\text { ansiedade, depressão e } \\
\text { sintomas } & \text { obsessivo- } \\
\text { compulsivos } & \text { em } \\
\text { profissionais de saúde e } \\
\text { outros profissionais } \\
\begin{array}{lr}\text { durante a pandemia } \\
\text { COVID-19. }\end{array} \\
\end{array}$ & $\begin{array}{l}\text { A análise demonstrou que os profissionais da } \\
\text { saúde eram significativamente mais propensos a } \\
\text { se preocuparem com sujeira, germes e vírus antes } \\
\text { da COVID-19, em relação aos demais } \\
\text { profissionais. Por outro lado, após o início da } \\
\text { pandemia, os demais profissionais demonstram-se } \\
\text { mais preocupados que os da saúde. O mesmo } \\
\text { padrão foi observado em relação ao } \\
\text { comportamento de lavagem compulsiva. }\end{array}$ \\
\hline Nissen et al. (2021) & Dinamarca & $\begin{array}{l}\text { Analisar o impacto da } \\
\text { COVID-19 em crianças e } \\
\text { adolescentes portadoras } \\
\text { de TOC. }\end{array}$ & $\begin{array}{l}\text { Os resultados apontaram uma piora dos sintomas } \\
\text { de TOC em ambos os grupos, sendo a dimensão } \\
\text { agressivo/sexual e o insight insatisfatório } \\
\text { preditores de gravidade do TOC e da piora dos } \\
\text { sintomas de ansiedade e depressão. Fatores como } \\
\text { a idade precoce e o histórico familiar de } \\
\text { Transtorno de Hiperatividade e Déficit de } \\
\text { Atenção (TDAH) foram associados à piora do } \\
\text { quadro clínico. }\end{array}$ \\
\hline Ornell et al. (2021) & Brasil & $\begin{array}{l}\text { Discutir o reforço do TOC } \\
\text { durante a pandemia de } \\
\text { COVID-19. }\end{array}$ & $\begin{array}{l}\text { Foi observado a necessidade de cuidar da saúde } \\
\text { mental da população e ressaltado que os pacientes } \\
\text { com TOC, principalmente aqueles na dimensão } \\
\text { limpeza, correm um risco potencialmente maior } \\
\text { de agravo dos sintomas durante a atual pandemia. }\end{array}$ \\
\hline
\end{tabular}




\begin{tabular}{|c|c|c|c|}
\hline Perkes et al. (2020) & Austrália & $\begin{array}{l}\text { Discutir a repercussão da } \\
\text { pandemia nos pacientes } \\
\text { com TOC, especialmente } \\
\text { nas compulsões de } \\
\text { contaminação. }\end{array}$ & $\begin{array}{l}\text { Foi apontado um impacto positivo inesperado } \\
\text { para a saúde mental em boa parte dos pacientes } \\
\text { com TOC de contaminação. Esses sentiram } \\
\text { seguros e validados em relação as suas } \\
\text { preocupações excessivas, por existirem atual- } \\
\text { mente rigorosas diretrizes sobre como lavar as } \\
\text { mãos. }\end{array}$ \\
\hline Prestia et al. (2020) & Itália & $\begin{array}{l}\text { Avaliar em pacientes com } \\
\text { TOC as mudanças nos } \\
\text { sintomas antes e durante a } \\
\text { quarentena. }\end{array}$ & $\begin{array}{l}\text { O artigo apontou uma piora nos sintomas dos } \\
\text { pacientes com TOC em relação ao período } \\
\text { anterior à pandemia. Os resultados mostraram que } \\
\text { indivíduos que não podiam trabalhar e/ou estudar } \\
\text { remotamente durante a quarentena, aqueles que } \\
\text { vivem com um dos pais na mesma casa e aqueles } \\
\text { com sintomas de contaminação tiveram uma } \\
\text { piora significativa na gravidade do transtorno. }\end{array}$ \\
\hline Rajkumar (2020) & Índia & $\begin{array}{l}\text { Discutir a relação da } \\
\text { pandemia de COVID-19 e } \\
\text { a evolução e origem do } \\
\text { transtorno obsessivo- } \\
\text { compulsivo. }\end{array}$ & $\begin{array}{l}\text { Foi possível observar evidência positiva e mais } \\
\text { convincente de uma associação entre algumas } \\
\text { dimensões de TOC e comportamentos que } \\
\text { minimizaram o risco de infecção. Os temores de } \\
\text { contaminação e lavagem ritualística } \\
\text { desempenham um papel na melhoria dos padrões } \\
\text { sanitários e da saúde em geral }\end{array}$ \\
\hline Rosa-Alcázar et al. (2021) & Espanha & $\begin{array}{l}\text { Comparar as estratégias } \\
\text { de enfrentamento utili- } \\
\text { zadas por pacientes com } \\
\text { transtorno obsessivo- } \\
\text { compulsivo (TOC) du- } \\
\text { rante o confinamento por } \\
\text { COVID-19 versus } \\
\text { controle saudável. }\end{array}$ & $\begin{array}{l}\text { O resultado indicou que os pacientes com TOC } \\
\text { apresentaram maiores médias em suporte } \\
\text { instrumental (busca de ajuda, aconselhamento e } \\
\text { informações de pessoas competentes), religião, } \\
\text { negação e autocensuras. Em contrapartida, o } \\
\text { grupo de controle saudável usou estratégias de } \\
\text { enfrentamento positivas como reenquadramento, } \\
\text { aceitação e humor. }\end{array}$ \\
\hline $\begin{array}{c}\text { Schwartz-Lifshitz et al. } \\
\text { (2021) }\end{array}$ & Israel & $\begin{array}{l}\text { Avaliar se crianças e } \\
\text { adolescentes com TOC } \\
\text { experimentaram exa- } \\
\text { cerbação dos sintomas } \\
\text { durante a pandemia de } \\
\text { COVID-19. }\end{array}$ & $\begin{array}{l}\text { No resultado, contrariando a hipótese dos autores, } \\
\text { não foi encontrado exacerbação e nem diferença } \\
\text { significativa na gravidade da doença, no ano } \\
\text { anterior à pandemia em comparação com o } \\
\text { período da pandemia. }\end{array}$ \\
\hline Shafran et al. (2020) & Inglaterra & $\begin{array}{l}\text { Reconhecer e abordar o } \\
\text { impacto do COVID-19 no } \\
\text { transtorno obsessivo- } \\
\text { compulsivo }\end{array}$ & $\begin{array}{l}\text { Foi observado que jovens e adultos foram os mais } \\
\text { suscetíveis ao desenvolver sintomas de TOC } \\
\text { durante a pandemia. Os mais afetados foram as } \\
\text { pessoas preocupadas em contaminarem ou } \\
\text { espalharem a doença, as com tendência de } \\
\text { verificação excessiva de notícias do COVID-19 e } \\
\text { as que superestimam as ameaças. }\end{array}$ \\
\hline Sharma et al. (2021) & Índia & $\begin{array}{l}\text { Avaliar o impacto da } \\
\text { pandemia COVID-19, a } \\
\text { curto prazo, nos pacientes } \\
\text { com TOC. }\end{array}$ & $\begin{array}{l}\text { O artigo obteve a conclusão de que a pandemia, } \\
\text { pelo menos a curto prazo, não influenciou o curso } \\
\text { do TOC. Conforme os resultados, os sintomas de } \\
\text { contaminação preexistentes e a ansiedade } \\
\text { relacionada à saúde, no contexto pandêmico } \\
\text { atual, não previram recidiva, no período avaliado. }\end{array}$ \\
\hline Silva et al. (2021) & Brasil & $\begin{array}{l}\text { Avaliar o impacto dos } \\
\text { pacientes com TOC na } \\
\text { pandemia de COVID-19. }\end{array}$ & $\begin{array}{l}\text { Concluiu que se espera um agravamento dos } \\
\text { sintomas de TOC em diferentes dimensões, como } \\
\text { contaminação/limpeza, agressão e acumulação. } \\
\text { Ainda relata que pacientes que nunca } \\
\text { apresentaram esses sintomas podem experimentar } \\
\text { seu início no contexto dessa grande mudança } \\
\text { ambiental }\end{array}$ \\
\hline Storch et al. (2021) & $\begin{array}{l}\text { Estados } \\
\text { Unidos }\end{array}$ & $\begin{array}{l}\text { Estimar o impacto geral } \\
\text { da pandemia de COVID- } \\
19 \text { nos sintomas dos } \\
\text { pacientes com TOC. }\end{array}$ & $\begin{array}{l}\text { A pesquisa constatou uma piora nos sintomas de } \\
\text { TOC durante a fase inicial da pandemia, sendo o } \\
\text { estresse financeiro e o risco de complicações pelo } \\
\text { vírus dois fatores significativamente prejudiciais. } \\
\text { Em contrapartida, foi observado que a interação } \\
\text { familiar desempenhou um papel importante na } \\
\text { diminuição da gravidade dos sintomas }\end{array}$ \\
\hline
\end{tabular}




\begin{tabular}{|c|c|c|c|}
\hline Tanir et al. (2020) & Turquia & $\begin{array}{l}\text { Analisar o impacto da } \\
\text { COVID-19 em pacientes } \\
\text { de TOC no grupo de } \\
\text { crianças e adolescentes. }\end{array}$ & $\begin{array}{l}\text { O estudo concluiu que houve um aumento na } \\
\text { gravidade dos sintomas no grupo em comento, } \\
\text { sendo as obsessões por contaminação e } \\
\text { compulsões de lavagem, os sintomas de TOC } \\
\text { mais frequentes antes e durante a pandemia. } \\
\text { Sendo constatado que variáveis como pouca } \\
\text { interação familiar, preocupação e pesquisas } \\
\text { diárias sobre o COVID-19, e o diagnóstico em } \\
\text { algum familiar foram significativas na gravidade } \\
\text { dos sintomas. }\end{array}$ \\
\hline Toh et al. (2021) & Austrália & $\begin{array}{l}\text { Comparar o estado de } \\
\text { saúde mental no grupo de } \\
\text { TOC em relação a um } \\
\text { grupo da população geral } \\
\text { em dois momentos: na } \\
\text { primeira onda (abril/2020) } \\
\text { e segunda onda } \\
\text { (maio/2020). }\end{array}$ & $\begin{array}{l}\text { Os resultados indicaram que na primeira onda, } \\
\text { pacientes com TOC apresentaram depressão, } \\
\text { ansiedade e estresse significativamente } \\
\text { aumentados em relação ao grupo da população } \\
\text { geral. Já na segunda onda, com o agravamento da } \\
\text { pandemia, esses resultados foram mais severos no } \\
\text { grupo de TOC e apenas moderados no grupo da } \\
\text { população geral. }\end{array}$ \\
\hline Tundo et al. (2021) & Itália & $\begin{array}{l}\text { Examinar em um ambi- } \\
\text { ente clínico o impacto do } \\
\text { estresse da COVID-19 em } \\
\text { pacientes ambulatoriais } \\
\text { com Depressão, } \\
\begin{array}{l}\text { Ansiedade ou TOC } \\
\text { preexistentes. }\end{array}\end{array}$ & $\begin{array}{l}\text { O artigo observou que as consequências do } \\
\text { estresse do COVID-19 nos pacientes avaliados } \\
\text { foram muito modestas. Portanto, não se pôde } \\
\text { confirmar a alta vulnerabilidade às consequências } \\
\text { diretas e indiretas do estresse pandêmico nos } \\
\text { pacientes com Ansiedade, Depressão ou TOC } \\
\text { pré-existentes. }\end{array}$ \\
\hline Wheaton et al. (2021) & $\begin{array}{l}\text { Estados } \\
\text { Unidos }\end{array}$ & $\begin{array}{l}\text { Investigar a relação entre } \\
\text { ansiedade excessiva e } \\
\text { sintomas de TOC durante } \\
\text { as fases iniciais da } \\
\text { pandemia de COVID-19. }\end{array}$ & $\begin{array}{l}\text { O artigo verificou correlações significativas entre } \\
\text { os sintomas de TOC e ansiedade e o medo da } \\
\text { propagação do COVID-19. Foi demostrado que } \\
\text { parte das ligações diretas entre o TOC e os } \\
\text { sintomas de ansiedade às preocupações } \\
\text { excessivas sobre o COVID-19 é a dificuldade em } \\
\text { tolerar a incerteza. }\end{array}$ \\
\hline Zhang et al. (2020) & China & $\begin{array}{l}\text { Avaliar o impacto da } \\
\text { pandemia de COVID-19 } \\
\text { nas manifestações } \\
\text { psicológicas em profis- } \\
\text { sionais de saúde. }\end{array}$ & $\begin{array}{l}\text { O estudo demonstrou que os profissionais de } \\
\text { saúde médicos e enfermeiros apresentaram } \\
\text { maiores taxas de prevalência de insônia, ansi- } \\
\text { edade, depressão, somatização e sintomas ob- } \\
\text { sessivo-compulsivos. Para esses últimos, foram } \\
\text { encontrados } 3 \text { fatores de risco: viver em áreas } \\
\text { rurais, estar em risco de contato com pacientes } \\
\text { COVID-19 em hospitais e ter doenças orgânicas. }\end{array}$ \\
\hline Zheng et al. (2020) & China & $\begin{array}{l}\text { Investigar a prevalência } \\
\text { de TOC na população } \\
\text { urbana de Wuhan durante } \\
\text { a fase de controle e } \\
\text { prevenção regular da } \\
\text { pandemia. }\end{array}$ & $\begin{array}{l}\text { O estudo constatou que a prevalência do TOC foi } \\
\text { maior grupo de jovens de } 15 \text { a } 24 \text { anos. E apesar } \\
\text { da pesquisa ser realizado em uma área segura, } 3 \\
\text { meses após o fim da quarentena, as pessoas em } \\
\text { ainda eram afetadas pelo TOC. Estudantes, } \\
\text { solteiros, portadores de transtornos mentais } \\
\text { comórbidos, história familiar de TOC e latência } \\
\text { do sono foram associados a maior prevalência do } \\
\text { diagnóstico de TOC }\end{array}$ \\
\hline
\end{tabular}

Fonte: Autores.

\section{Discussão}

No presente trabalho, conforme já mencionado, foram analisados 30 artigos, dos quais 16 estudos (Aardena et al. (2020), Benatti et al. (2020), Jelinek et al. (2021), Kaveladze et al. (2021), Khosravani (2021a, 2021b), Nissen; Hojgaard e Thomsen (2020), Ornell et al. (2021), Prestia et al. (2020), Rosa-Alcázar et al. (2021), Shafran et al. (2020), Silva et al. (2021), Storch et al. (2021), Tanir et al. (2020), Toh et al. (2021), Wheaton et al. (2021)) verificaram exacerbação e alteração na gravidade dos sintomas de TOC em pacientes com diagnostico prévio do transtorno, durante a pandemia de COVID-19 e 5 artigos (Abba-Aji et al. (2020), Ji Guangjun et al. (2020), Mrklas et al. (2020), Zhang et al. (2020), Zheng et al. (2020) observaram aumento de sintomas obsessivos-compulsivos na população geral.

Em contrapartida, 9 pesquisas (Alhusseini et al. (2021), Chakraborty \& Karmakar (2020), Littman et al. (2020), 
Matsunaga; Mukai e Yamanishi (2020), Perkes et al. (2020), Rajkumar (2020), Schwartz-Lifshitz et al. (2021), Sharma et al. (2021), Tundo et al. (2021)) apontaram que o aumento de sintomas em pacientes com TOC foi indiferente ou insignificante frente à pandemia.

Das publicações supracitadas que identificaram a exacerbação dos sintomas, o agravo nas obsessões de contaminação e compulsões de lavagem teve uma maior evidência, tanto nos que já possuíam essas condições quanto naqueles que mudaram de fenótipo ou apresentaram novos sintomas. De acordo com os resultados, o contexto pandêmico atual de COVID-19 trouxe implicações significativas ou até drásticas para esses pacientes.

Vale ressaltar ainda que o agravo dos sintomas de TOC foi também relatado em pacientes que apresentam outras dimensões da doença, sendo percebido o aumento de compulsões de evitação, verificação, acumulação e agressividade. De acordo com Benatti et al. (2020), houve um crescimento das taxas de comportamentos de evitação, principalmente relacionados ao medo de uma possível contaminação. Já Shafran et al. (2020) destacam que a verificação excessiva de notícias e eventos relacionados ao COVID-19 pode resultar em maiores preocupações sobre a responsabilidade por danos e verificação de comportamentos.

Como justificam Ornell et al. (2020), o risco iminente de contaminação cria uma necessidade lógica de autovigilância e hábitos de higiene, prejudicial a indivíduos com TOC, uma vez que distorções cognitivas e estratégias compensatórias (rituais de limpeza) não são mais irracionais ou superdimensionadas, pelo contrário, essas ideias tornam-se legítimas e socialmente aceitas, gerando validação plausível para a intensificação da compulsão e rituais de limpeza.

Segundo Benatti et al (2020) a exacerbação dos sintomas nesses pacientes pode estar relacionada à necessidade de maior controle contra contaminação potencial ou ao aumento do tempo livre durante a pandemia, levando a um crescimento de comportamentos repetitivos. Além disso, os padrões de higiene que se tornaram mais rigorosos durante a pandemia, apesar de se tratarem de medidas simples e parecerem fáceis de seguir, podem afetar pacientes de TOC de forma mais onerosa, já que atacam sua insegurança quanto a medidas de higiene e necessidade limpeza compulsiva.

Ainda a despeito dos pacientes de TOC nas dimensões de contaminação e lavagem, uma problemática foi identificada; como aduziram Silva et al. (2021), as técnicas fundamentais utilizadas no tratamento do TOC, como a exposição e a prevenção ritual, não podem ser utilizadas de forma ilimitada, tendo em vista que recomendar a exposição irrestrita a estímulos temidos, nesse período pandêmico, pode ser imprudente, comprometendo a saúde e segurança dos pacientes.

Convém destacar que parte das pesquisas relacionou o aumento dos sintomas de pacientes com TOC no período pandêmico ao aumento de estresse, ansiedade e depressão. Conforme apontou o estudo de Matsunaga et al. (2020), indivíduos com sintomas de TOC gravemente afetados por COVID-19 foram significativamente mais propensos a ter maior traço de ansiedade, estado depressivo, e maior prevalência de transtorno de ansiedade generalizada. Corroborando com esses achados, Littman et al. (2020) concluíram que os pacientes que apresentaram pioram sintomática relataram mais sentimentos de ansiedade e estresse e embora essa causalidade não pôde ser inequivocamente inferida a partir dos seus achados, isso sugeriu que a ansiedade e o estresse podem predizer a reação de alguém à crise COVID-19.

Quanto à dimensão de agressividade, Nissen et al. (2021) referem que raiva e atitudes pessimistas e sintomas agressivos em relação ao futuro incerto, causado pela pandemia, podem resultar em um aumento dos sintomas envolvendo temas imorais, sexuais, suspeitos e violentos. Já no caso da acumulação, Rajkumar (2020) constatou que os pacientes com esse tipo de TOC acumulam materiais que são de pouco ou nenhum uso objetivo para eles; por outro lado, durante a atual pandemia, materiais que são objetivamente úteis (máscaras, desinfetantes) estão sendo acumulados, mas a uma extensão que é inadequada e excessiva.

Um ponto controverso visto nos estudos foi a variável de interação familiar durante a pandemia de COVID-19 nos pacientes com TOC. Enquanto que algumas pesquisas constataram que os pacientes com TOC que tinham interação familiar 
durante a pandemia desenvolveram menos sintomas, outros artigos indicaram que a interação familiar para esses pacientes foi um agravante desencadeador de acomodação familiar. Nesse sentido, frisa-se o achado de Prestia et al. (2020), que indicou que morar com um parente na mesma casa pode ser um fator de proteção (ou seja, através do suporte emocional fornecido por um parente durante a quarentena) ou um fator de vulnerabilidade para o agravamento dos sintomas (ou seja, aumento do risco de acomodação familiar ou emoção expressa).

Contrapondo-se à maioria dos estudos que apontaram a exacerbação de efeitos negativos nos pacientes com TOC, algumas pesquisas não revelaram aumento de sintomas em grupos de pacientes com TOC, cuja mudança foi indiferente ou insignificante frente à pandemia. Nesse ínterim, o trabalho de Sharma et al. (2021) obteve um resultado indiferente, os sintomas de contaminação preexistentes e a ansiedade decorrente do contexto pandêmico atual não previram recidiva, contudo, há a ressalva de ser uma análise restrita apenas a curto prazo.

Na pesquisa de Chakraborty e Karmakar (2020), conduzida num grupo de pacientes com TOC, particularmente com obsessão por contaminação e compulsão por lavar, também não foi percebido um aumento significativo dos sintomas, haja vista que a maioria dos pacientes pontou quase o mesmo na escala Y-BOCS, não mostrando qualquer aumento em seu comportamento de lavar.

Para Jelinek et al. (2021), as razões potenciais para a diminuição nos sintomas de TOC durante a pandemia de COVID-19 podem ser decorrentes da diminuição na estigmatização e vergonha (por exemplo, devido a medidas tomadas para prevenção de infecções, aumento do medo de contaminação na população em geral) e do fortalecimento das crenças funcionais. Além disso, as pessoas com TOC (particularmente aquelas com compulsão por lavar) também podem até experimentar um senso de competência como resultado de suas crenças funcionais e do uso de estratégias de enfrentamento adaptativas.

Outras pesquisas focaram a análise do impacto da COVID-19 em pacientes de TOC compreendidos apenas no grupo de crianças e adolescentes. Desses artigos, a maioria também verificou exacerbação dos sintomas e alteração na gravidade de TOC nesse grupo. No estudo de Tanir et al. (2020), foi evidenciado que as obsessões por contaminação e compulsões por limpar/lavar foram os sintomas de TOC mais frequentes nessa população tendo um aumento significativo durante a pandemia, e as variáveis como pouca interação com os familiares, preocupação diária com COVID-19, pesquisas excessivas sobre essa doença e diagnóstico em algum familiar, geraram mudanças significativas na gravidade dos sintomas.

Já no artigo de Nissen et al. (2021) foi constatado que os sintomas de TOC agressivo/sexual e o insight insatisfatório no grupo de crianças e adolescentes foram preditores de um pior resultado tanto na gravidade do TOC quanto na piora dos sintomas de ansiedade e depressão. Segundo esses autores, as inúmeras reportagens diárias destacando gravidade da doença e números de mortes pela COVID-19 tornam esse grupo mais afetado por pensamentos agressivos ou preocupações quanto à perda de seus cuidadores principais. Ademais, o insight insatisfatório implica um grau de incerteza de uma ameaça potencial, dessa forma, as crianças e os adolescentes têm maio vulnerabilidade, portanto, estão mais propensos a reagir com mais preocupação e medo.

Entretanto, contrapondo-se a esses resultados, no trabalho de Schwartz-Lifshitz et al (2021) não foi encontrada exacerbação e nem diferença significativa na gravidade do TOC no grupo de crianças e adolescentes, indicando até uma possível melhora durante a quarentena relacionada à redução das expectativas e menor envolvimento com fatores como a escola e a interação social. De acordo com o artigo, crianças e adolescentes com TOC lidam com fardos subjetivos, como tristeza e medo de contaminação, mas, ao contrário dos adultos, não são incomodados por fardos objetivos, como perda de emprego, problemas econômicos e um senso de propósito diminuído. Além disso a diminuição das demandas funcionais, comportamentais e sociais decorrentes do fechamento da escola, podem ter diminuído os níveis de estresse e contribuído para o curso favorável do TOC durante a pandemia de COVID-19. 
Outro grupo que foi alvo das pesquisas em relação ao TOC e os efeitos da atual pandemia foram os profissionais da saúde, no estudo científico de Zhang et al. (2020) foi constatado maior endosso de sintomas de TOC em trabalhadores médicos em relação aos demais profissionais da saúde. No estudo, foi percebido que os profissionais da área de saúde apresentaram maiores taxas de prevalência de sintomas obsessivo-compulsivos, insônia, ansiedade, depressão e somatização, sendo identificado três fatores de risco, quais sejam, viver em áreas rurais, estar em risco de contato com pacientes COVID-19 em hospitais e ter doenças orgânicas.

No estudo de Mrklas et al. (2020) a abordagem realizada entre os profissionais de saúde e os demais trabalhadores, demonstrou que os profissionais de saúde eram significativamente mais propensos a se preocupar com sujeira, germes vírus e apresentar comportamento de lavagem compulsiva antes da COVID-19 em comparação com outros trabalhadores. Por outro lado, após o início da pandemia, a proporção de preocupação dos profissionais de saúde demostrou-se menor em relação ao demais grupos. Segundo os autores, uma possível explicação para inversão é o fato desses estarem mais protegidos de danos à saúde mental devido à influência de outros modificadores documentados, como os seguintes: acesso imediato a conhecimentos relevantes, treinamento, protocolos, informações oportunas, equipamento de proteção individual e uma rede de apoio de colegas experimentando estresse semelhante.

Paralelamente, alguns estudos se dirigiram não aos pacientes com TOC especificamente, mas a uma abordagem na população geral para avaliar o impacto da pandemia de COVID-19 nas manifestações psicológicas. Para Shafran et al. (2020), até mesmo as pessoas sem preocupações e doenças pré-existentes podem inadvertidamente cair na armadilha da lavagem compulsiva das mãos se repetirem o estereótipo e o processo cronometrado de lavagem das mãos preconizado durante a crise, como forma de proporcionar alívio da ansiedade.

Corroborando com esse entendimento, a pesquisa de Abba-Aji et al. (2020), realizada sob a análise da população geral, observou que a maioria da amostragem desenvolveu obsessões relacionadas à contaminação e compulsões de lavagens, ambas iniciadas durante a pandemia, e esses indivíduos que relataram essas novas obsessões eram mais vulneráveis a transtorno de ansiedade generalizada e transtorno depressivo.

Por fim, percebendo a ampla diversidade de abordagens sobre o TOC na pandemia, Rosa-Alcázar et al. (2021), focaram sua pesquisa nas estratégias de enfrentamento ao período pandêmico, especialmente nos pacientes com TOC. O resultado indicou que os pacientes com TOC apresentaram maiores médias em suporte instrumental e de aconselhamento, religião, negação e autocensura, que podem estar relacionadas às próprias obsessões e compulsões, especificamente, com as obsessões de verificação, culpa, controle e compulsões religiosas. E essa estratégia de enfrentamento em excesso pode ser contraproducente para esses pacientes e exacerbar comportamentos obsessivos e de ansiedade. Por outro lado, o grupo de controle saudável usou estratégias de enfrentamento positivas como reenquadramento, aceitação e humor.

\section{Conclusão}

A pandemia de COVID-19 está afetando todos os âmbitos das relações humanas, gerando um grande impacto na saúde mental da população global e consequentemente. nos pacientes com transtorno obsessivo-compulsivo (TOC). Através dessa revisão de literatura, concluiu-se que, nesse período de COVID-19, a maioria das pesquisas indicou a exacerbação e alteração na gravidade dos sintomas em pacientes com TOC, especialmente naqueles que sofrem obsessão de contaminação e compulsão de lavagem.

Essa revisão também apontou o agravamento dos sintomas em outras dimensões do TOC, sendo observada a piora em paciente com comportamentos de verificação, evitação, agressividade e acumulação. Além disso, os resultados identificaram que os pacientes que apresentaram uma piora dos sintomas de forma geral demonstraram um aumento dos sintomas de estresse, ansiedade e depressão. E isso pode ser explicado porque o período pandêmico leva ao isolamento social, à alteração na rotina e 
à ociosidade, além da propagação global inesgotável de notícias acerca da doença e seu desenvolvimento no mundo.

Entretanto, cumpre destacar que alguns estudos não evidenciaram essa piora clínica nos pacientes com TOC, e essas divergências encontradas podem ser explicadas pelas variáveis utilizadas nas pesquisas, como localização, estágio da pandemia, duração da quarentena, nível socioeconômico e a amostragem.

Por fim, também foi observado o aumento de sintomas obsessivos compulsivos na população geral, especialmente relacionados à contaminação e lavagem, haja que vista que o enfrentamento do quadro pandêmico exige normas rigorosas de limpeza e higiene. Dessa forma, fazem-se necessárias pesquisas futuras pós Covid-19 para avaliar como evoluirá o quadro clínico dos pacientes que apresentaram exacerbação do TOC, bem como para determinar se os novos sintomas de TOC na população geral estão relacionados a um diagnóstico definitivo de TOC, a uma expressão de ansiedade proporcionada pela pandemia ou a uma combinação de ambos.

\section{Referências}

Abba-Aji, A., Li, D., Hrabok, M., Shalaby, R., Gusnowski, A., Vuong, W., Surood, S., Nkire, N., Li, X.-M., Greenshaw, A. J., \& Agyapong, V. I. O. (2020). COVID-19 pandemic and mental health: Prevalence and correlates of new-onset obsessive-compulsive symptoms in a Canadian province. International Journal of Environmental Research and Public Health, 17(19), 6986. https://doi.org/10.3390/ijerph17196986.

AlHusseini, N., Sajid, M., Altayeb, A., Alyousof, S., Alsheikh, H., Alqahtani, A., \& Alsomali, A. (2021). Depression and obsessive-compulsive disorders amid the COVID-19 pandemic in Saudi Arabia. Cureus, 13(1), e12978. Benatti, B. et al. (2020). What Happened to Patients With Obsessive Compulsive Disorder During the COVID-19 Pandemic? A Multicentre Report From Tertiary Clinics in Northern Italy. Frontiers in Psychiatry, 11. https://doi.org/10.3389/fpsyt.2020.00720.

Chakraborty, A., \& Karmakar, S. (2020). Impact of COVID-19 on Obsessive Compulsive Disorder (OCD). Iranian Journal of Psychiatry. https://doi.org/10.18502/ijps.v15i3.3820.

Jelinek, L., Moritz, S., Miegel, F., \& Voderholzer, U. (2021). Obsessive-compulsive disorder during COVID-19: Turning a problem into an opportunity? Journal of Anxiety Disorders, 77, 102329. https://doi.org/10.1016/j.janxdis.2020.102329.

Ji, G., Wei, W., Yue, K.-C., Li, H., Shi, L.-J., Ma, J.-D., He, C.-Y., Zhou, S.-S., Zhao, Z., Lou, T., Cheng, J., Yang, S.-C., \& Hu, X.-Z. (2020). Effects of the COVID-19 pandemic on obsessive-compulsive symptoms among university students: Prospective cohort survey study. Journal of Medical Internet Research, 22(9), e21915. https://doi.org/10.2196/21915.

Kaveladze, B., Chang, K., Siev, J., \& Schueller, S. M. (2021). Impact of the COVID-19 Pandemic on Online Obsessive-Compulsive Disorder Support Community Members: Survey Study. JMIR Mental Health, 8(2), e26715. https://doi.org/10.2196/26715.

Khosravani, V., Aardema, F., Samimi Ardestani, S. M., \& Sharifi Bastan, F. (2021a). The impact of the coronavirus pandemic on specific symptom dimensions and severity in OCD: A comparison before and during COVID-19 in the context of stress responses. Journal of Obsessive-Compulsive and Related Disorders, 29, 100626. https://doi.org/10.1016/j.jocrd.2021.100626.

Khosravani, V., Asmundson, G. J. G., Taylor, S., Sharifi Bastan, F., \& Samimi Ardestani, S. M. (2021b). The Persian COVID stress scales (Persian-CSS) and COVID-19-related stress reactions in patients with obsessive-compulsive and anxiety disorders. Journal of Obsessive-Compulsive and Related Disorders, 28 , 100615. https://doi.org/10.1016/j.jocrd.2020.100615.

Littman, R., Naftalovich, H., Huppert, J. D., \& Kalanthroff, E. (2020). Impact of COVID -19 on obsessive-compulsive disorder patients. Psychiatry and Clinical Neurosciences, 74(12), 660-661. https://doi.org/10.1111/pcn.13152.

Matsunaga, H., Mukai, K., \& Yamanishi, K. (2020). Acute impact of COVID -19 pandemic on phenomenological features in fully or partially remitted patients with obsessive-compulsive disorder. Psychiatry and Clinical Neurosciences, 74(10), 565-566. https://doi.org/10.1111/pcn.13119.

Mrklas, K., Shalaby, R., Hrabok, M., Gusnowski, A., Vuong, W., Surood, S., Urichuk, L., Li, D., Li, X.-M., Greenshaw, A. J., \& Agyapong, V. I. O. (2020). Prevalence of perceived stress, anxiety, depression, and obsessive-compulsive symptoms in health care workers and other workers in Alberta during the COVID-19 pandemic: Cross-sectional survey. JMIR Mental Health, 7(9), e22408. https://dx.doi.org/10.2196\%2F22408.

Nissen, J. B., Højgaard, D. R. M. A., \& Thomsen, P. H. (2020). The immediate effect of COVID-19 pandemic on children and adolescents with obsessive compulsive disorder. BMC Psychiatry, 20(1). https://doi.org/10.1186/s12888-020-02905-5.

Ornell, F., Braga, D. T., Bavaresco, D. V., Francke, I. D., Scherer, J. N., von Diemen, L., \& Kessler, F. H. P. (2021). Obsessive-compulsive disorder reinforcement during the COVID-19 pandemic. Trends in Psychiatry and Psychotherapy. https://doi.org/10.47626/2237-6089-2020-0054.

Pereira, A. S., Shitsuka D. M., Parreira F. J., Shitsuka R. (2018). Metodologia da Pesquisa Científica (1 ${ }^{\text {a }}$ ed.). UFSM, NTE. Disponivel em: https://www.ufsm.br/app/uploads/sites/358/2019/02/Metodologia-da-Pesquisa-Cientifica_final.pdf.

Perkes, I., Brakoulias, V., Lam-Po-Tang, J., Castle, D., \& Fontenelle, L. (2020). Contamination compulsions and obsessive-compulsive disorder during COVID-19. Australian \& New Zealand Journal of Psychiatry, 54(11), 1137-1138. https://doi.org/10.1177/0004867420952846. 
Davide, P., Andrea, P., Martina, O., Andrea, E., Davide, D., \& Mario, A. (2020). The impact of the COVID-19 pandemic on patients with OCD: Effects of contamination symptoms and remission state before the quarantine in a preliminary naturalistic study. Psychiatry Research, 291 , 113213. https://doi.org/10.1016/j.psychres.2020.113213.

Rajkumar, R. P. (2020). Contamination and infection: What the coronavirus pandemic could reveal about the evolutionary origins of obsessive-compulsive disorder. Psychiatry Research, 289, 113062. https://doi.org/10.1016/j.psychres.2020.113062.

Rosa-Alcázar, Á., García-Hernández, M. D., Parada-Navas, J. L., Olivares-Olivares, P. J., Martínez-Murillo, S., \& Rosa-Alcázar, A. I. (2021). Coping strategies in obsessive-compulsive patients during Covid-19 lockdown. International Journal of Clinical and Health Psychology, 21(2), 100223. https://doi.org/10.1016/j.ijchp.2021.100223.

Schwartz-Lifshitz, M., Basel, D., Lang, C., Hertz-Palmor, N., Dekel, I., Zohar, J., \& Gothelf, D. (2021). Obsessive compulsive symptoms severity among children and adolescents during COVID-19 first wave in Israel. Journal of Obsessive-Compulsive and Related Disorders, 28(100610), 100610. https://dx.doi.org/10.2196\%2F22408.

Shafran, R., Coughtrey, A., \& Whittal, M. (2020). Recognising and addressing the impact of COVID-19 on obsessive-compulsive disorder. The Lancet Psychiatry, 7(7), 570-572. https://doi.org/10.1016/s2215-0366(20)30222-4.

Sharma, L. P., Balachander, S., Thamby, A., Bhattacharya, M., Kishore, C., Shanbhag, V., Sekharan, J. T., Narayanaswamy, J. C., Arumugham, S. S., \& Reddy, J. Y. C. (2021). Impact of the COVID-19 pandemic on the short-term course of obsessive-compulsive disorder. The Journal of Nervous and Mental Disease, 209(4), 256-264. https://doi.org/10.1097/nmd.0000000000001318.

Silva, R. M., Shavitt, R. G., \& Costa, D. L. (2021). Obsessive-compulsive disorder during the COVID-19 pandemic. Brazilian Journal of Psychiatry, 43(1), 108-108. https://doi.org/10.1590/1516-4446-2020-1189.

Storch, E. A., Sheu, J. C., Guzick, A. G., Schneider, S. C., Cepeda, S. L., Rombado, B. R., Gupta, R., Hoch, C. T., \& Goodman, W. K. (2021). Impact of the COVID-19 pandemic on exposure and response prevention outcomes in adults and youth with obsessive-compulsive disorder. Psychiatry Research, 295(113597), 113597. https://doi.org/10.1016/j.psychres.2020.113597.

Tanir, Y., Karayagmurlu, A., Kaya, İ., Kaynar, T. B., Türkmen, G., Dambasan, B. N., Meral, Y., \& Coşkun, M. (2020). Exacerbation of obsessive compulsive disorder symptoms in children and adolescents during COVID-19 pandemic. Psychiatry Research, 293(113363), 113363. https://doi.org/10.1016/j.psychres.2020.113363.

Toh, W. L., Neill, E., Phillipou, A., Tan, E. J., Van Rheenen, T. E., Meyer, D., \& Rossell, S. L. (2021). Obsessive-compulsive disorder and related symptoms amidst the COVID-19 outbreak: Results from the COLLATE project. Psychiatry and Clinical Neurosciences, 75(4), 146148. https://doi.org/10.1111/pcn.13202.

Tundo, A., Betro', S., \& Necci, R. (2021). What Is the Impact of COVID-19 Pandemic on Patients with Pre-Existing Mood or Anxiety Disorder? An Observational Prospective Study. Medicina, 57(4), 304. https://doi.org/10.3390/medicina57040304.

Wheaton, M. G., Messner, G. R., \& Marks, J. B. (2021). Intolerance of uncertainty as a factor linking obsessive-compulsive symptoms, health anxiety and concerns about the spread of the novel coronavirus (COVID-19) in the United States. Journal of Obsessive-Compulsive and Related Disorders, $28,100605$. https://doi.org/10.1016/j.jocrd.2020.100605.

Zhang, W.-R., Wang, K., Yin, L., Zhao, W.-F., Xue, Q., Peng, M., Min, B.-Q., Tian, Q., Leng, H.-X., Du, J.-L., Chang, H., Yang, Y., Li, W., Shangguan, F.-F., Yan, T.-Y., Dong, H.-Q., Han, Y., Wang, Y.-P., Cosci, F., \& Wang, H.-X. (2020). Mental health and psychosocial problems of medical health workers during the COVID-19 epidemic in China. Psychotherapy and Psychosomatics, 89(4), 242-250. https://doi.org/10.1159/000507639.

Zheng, Y., Xiao, L., Xie, Y., Wang, H., \& Wang, G. (2020). Prevalence and Characteristics of Obsessive-Compulsive Disorder Among Urban Residents in Wuhan During the Stage of Regular Control of Coronavirus Disease-19 Epidemic. Frontiers in Psychiatry, 11. https://doi.org/10.3389/fpsyt.2020.594167. 